\title{
Aproximación a un protocolo quirúrgico para el manejo de dientes supernumerarios: Evaluación de un caso y revisión de la literatura
}

\section{Olate Morales S*, Alister Herdener JP, Thomas Maldonado D, Alveal Campuzano R}

\section{RESUMEN}

Introducción: Los dientes supernumerarios (DSN) son anomalías del desarrollo, cuyas manifestaciones típicas suelen ser modificaciones en secuencia y erupción dentaria.

Metodología: Se realizo una búsqueda en Medline y Pubmed con criterios de selección definidos entre los años 1985 y 2005.

Resultados: Se encontraron 13 artículos útiles para la revisión, de los cuales 2 fueron revisiones bibliográficas. Discusión: La prevalencia de DSN rodea al $2 \%$, presentándose principalmente en hombres. No siempre la exéresis de DSN es necesaria por lo que se debe conocer claramente sus indicaciones además de presentar un estudio radiográfico previo detallado. El protocolo quirúrgico exige una descripción y clasificación del DSN para planificar su tratamiento en un equipo multidisciplinario. Su tratamiento quirúrgico presenta generalmente un pronóstico favorable y sus complicaciones se asocian con cierta frecuencia a las estructuras anatómicas vecinas. Conclusión: Este protocolo quirúrgico ayuda a sistematizar los procedimientos terapéuticos para DSN y hacerlos mas predecibles.

Palabras clave: Diente supernumerario, Cirugía oral, Protocolo quirúrgico.

\section{SUMMARY}

Introduction: The supernumerary teeth (DSN) are development anomalies, which typical manifestations are sequence and eruption modifications.

Methodology: We did a search in Medline and Pubmed with definied criteria of selection between the year 1985 and 2005.

Results: 13 articles were found useful for the review, of which 2 were literature reviews.

Discussion: DSN's prevalence surrounds $2 \%$, appearing principally between men. Not always DSN's exodontia is necessary for what must know clearly his indications and presenting a radiographic previous study. The surgical protocol demands a description and classification of the DSN to plan the treatment in a multidisciplinary team. The surgical treatment presents generally a favorable prediction and it's complications are associated with certain frequency to the anatomical neighboring.

Conclusion: This surgical protocol helps to systematize the therapeutic procedures for DSN and to do them more predecible.

Key words: Supernumerary Tooth, Oral Surgery, Surgical Protocol.

Fecha de recepción: Noviembre 2006.

Aceptado para publicación: Diciembre 2006.

* Cirujano-Dentista. Departamento de Odontología Integral. Facultad de Medicina. Universidad de La Frontera. Temuco, Chile.

Olate Morales S, Alister Herdener JP, Thomas Maldonado D, Alveal Campuzano R. Aproximación a un protocolo quirúrgico para el manejo de dientes supernumerarios: Evaluación de un caso y revisión de la literatura. Av. Odontoestomatol 2007; 23 (2): 67-73. 


\section{INTRODUCCIÓN}

Los dientes supernumerarios (DSN) corresponden a una de las alteraciones del desarrollo que acompañan al hombre desde hace ya mucho tiempo. De hecho, su presencia fue reportada en un fósil de Australopithecus de 1,7 millones de años (1).

Los pacientes con DSN exigen de los clínicos un tratamiento multidisciplinario, donde deben interactuar ortodoncistas, odontopediatras y cirujanos orales; con esto se logra una correcta planificación para lograr soluciones exitosas.

La epidemiología de los DSN es controvertida. Bäckman y cols. (2) aportaron que estos casos representaban el 1,9\% de los niños suecos de 7 años, donde hubo más casos en mujeres que en hombres, lo que se contradice con el estudio de Patchett (3) y Mason (4), quienes señalaron que la relación hombre-mujer en estas patologías es aproximadamente $2: 1$. Los dientes supernumerarios también se asocian más frecuentemente a maxilar anterior zona anterior del maxilar superior (5) y a la dentición permanente. Betts (6) señaló que la causa más común de incisivos centrales no erupcionados era la presencia de dientes supernumerarios, aportando que el $47 \%$ de los sujetos en tal condición presentan al menos un diente extra.

La intervención quirúrgica en estos casos exige consideraciones especiales, ya que se presentan diferentes variables a las de una exodoncia común, ya sea por la ubicación, estado y edad del paciente, etc. (7).

Algunos estudios han revelado la ausencia de protocolos de tratamiento para los pacientes que presentan DSN, determinando resultados integrales poco predecibles (3).

Al identificar un DSN, gran parte de los odontólogos opta por la exéresis inmediata, sin definir sus condiciones, etapas claves, complicaciones postoperatorias, etc. Es por ello, que nuestro equipo ha trabajado desde ya algún tiempo en definir protocolos quirúrgicos que permitan optimizar y hacer predecibles los tratamientos. Por ello, el objetivo de esta revisión es utilizar la información actual acerca de los DSN para definir un protocolo quirúrgico para el manejo de DSN.

\section{METODOLOGÍA}

Se realizo una búsqueda en PubMed y Medline de publicaciones en journals odontológicos. Las palabras de búsqueda fueron "supernumerary tooth", "supplemental tooth" y "unerupted tooth". La búsqueda se detallo a publicaciones entre 1985 y 2005 que fuesen escritas en ingles, correspondiendo a estudios de casos y controles, de cohorte, reportes clínicos y revisiones bibliográficas. Finalmente, se desarrollaron casos clínicos, de los cuales presentamos uno en esta publicación.

\section{RESULTADOS}

Encontramos 13 publicaciones que pudiesen servir para cumplir nuestro objetivo. De ellas, dos correspondieron a revisiones bibliográficas. De las 11 restantes, la mayoría fueron estudios de casos y estudios retrospectivos. Con esta información se desarrolló el protocolo de manejo de DSN.

\section{PROTOCOLO DE INTERVENCIÓN QUIRÚRGICA}

\section{Diagnóstico}

El diagnóstico de dientes supernumerarios se puede dar en diferentes circunstancias, siendo principalmente de dos formas $(8,9)$ :

1. Hallazgo radiográfico, donde el paciente no presenta ningún signo que refleje su presencia.

2. El paciente presenta alguna alteración cuya causa probable es la presencia de un diente supernumerario; estas situaciones pueden ser:

- Dolor en la zona, reflejada como "presión en el diente", en ausencia de caries, periodontitis u otra patología clínicamente visible.

- Ausencia de la pieza dentaria cuando la pieza contralateral ya se encuentra erupcionada.

- Mal posición de una pieza dentaria (rotaciones, inclinaciones, etc.).

El examen complementario radiográfico debe ser riguroso y exhaustivo, otorgándole gran importancia a la interpretación que realice el radiólogo maxilofacial. 
Algunos estudios han evaluado la necesidad de una o mas radiografías y cuales son las más necesarias. Patchett (3) concluye que el apoyo radiográfico se debería evaluar con una radiografía céntrica periapical, una excéntrica periapical, una panorámica o bien una lateral de cráneo, ya que en las radiografías oclusales existen muchas complicaciones al observar estas estructuras (10).

No obstante, en un estudio retrospectivo realizado por Mason (4) se describió que el $91 \%$ de los diagnósticos se realizo con radiografía oclusal, $85 \%$ con radiografías panorámicas, $54 \%$ con radiografías periapicales y $23 \%$ con radiografías laterales.

La decisión de la radiografía requerida siempre pasara por la necesidad del clínico, pero siempre deberían existir al menos dos radiografías con distinta angulación (4). Con estas placas se puede identificar la posición, la forma y las características generales de la estructura. De esta forma, las técnicas de paralelismo son las de elección para localizar dientes no erupcionados anteriores o posteriores (10).

\section{Clasificación}

La clasificación es necesaria para considerar parte de los aspectos de la probable intervención quirúrgica. Existen distintas clasificaciones algunas de las cuales discrepan, incluyendo los odontomas como dientes supernumerarios ya que insertan la presencia de odontomas como supernumerarios (8). Nosotros utilizaremos la clasificación propuesta por Rusell (9), excluyendo la presencia de odontomas. De esta forma, los dientes supernumerarios son:

1. Singles: $68 \%$ (4)

- Cónicos: son los que presentan solo una cúspide y generalmente son únicos. $64 \%$ de los pacientes con mesiodens (3). Generalmente presenta estructura similar a un diente con formación radicular completa o parcial.

- Tuberculado: presentan más de una cúspide. $36 \%$ de los pacientes con mesiodens (3). Generalmente su ubican en palatino de los dientes anterosuperiores.
2. Múltiples: $32 \%$ (4)

- Asociado a Síndrome.

- Suplementario: Duplicación de un diente en su serie normal, generalmente asociado a la dentición permanente.

Cuando los dientes extras han sido diagnosticados y clasificados, se debe evaluar su necesidad de tratamiento. Algunos requieren intervención quirúrgica, mientras otros no. Además es importante establecer cuando se debe realizar la intervención $(8,9)$.

\section{Intervención quirúrgica}

\section{Indicación para la remoción quirúrgica (8)}

1. Erupción del incisivo central es alterada o inhibida.

2. Desplazamiento evidente de los incisivos centrales.

3. Asociación de patologías al diente supernumerario.

4. Tratamiento ortodóncico de dientes asociados a dientes supernumerarios.

5. En casos de injerto óseo en paladar fisurado u otras patologías.

6. En situaciones en que el reborde es indicado para implante.

7. Cuando existe erupción espontánea del diente supernumerario.

\section{Momento de la intervención}

Existe información variable en cuanto a cual es el mejor momento de intervención quirúrgica. Previos estudios han señalado que la edad promedio de remoción quirúrgica del diente supernumerario es 9 años 4 meses (3). Sin embargo, existen dos situaciones para evaluar: la primera de ellas muestra la prontitud de la extracción del diente según las complicaciones mediatas e inmediatas del mismo (dolor, complicaciones ortodóncicas, etc.); la segunda está dada por la ausencia de tales complicaciones de modo que considerar el estado del desarrollo radicular de los dientes permanentes y del supernumerario es importante previo a la toma de decisiones. 
La erupción del diente retenido se vería complicada cuando este ya se encuentre con formación radicular completa, de forma que la intervención quirúrgica previa sería necesaria (4). Así, luego de la remoción del diente supernumerario mientras mas incompleta es la formación radicular del diente no erupcionado, existe mayor posibilidad que erupcione espontáneamente (4).

Algunos autores son partidarios del manejo conservador (5). Lo cierto es que la remoción quirúrgica inmediata se indicara cuando exista alguna asociación patológica o estética que sea originada por el diente supernumerario (11).

\section{Complicaciones y pronóstico}

Mantener un diente supernumerario en boca puede significar la aparición de patologías mayores, como aumentos de volumen y dolor (12), alteraciones en la sensibilidad por compresión de nervios (13), retención del diente permanente (4), y otros. La exodoncia de este se decidirá en base a la planificación del caso con un equipo multidisciplinario (ortodoncista, odontopediatra, radiólogo y cirujano) a fin de definir criterios y escoger la mejor alternativa junto al paciente y su familia.

El pronóstico de estos pacientes una vez retirado el diente supernumerario es bueno (6), ya que se elimina el factor desencadenante de las complicaciones. No obstante, futuros tratamientos ortodóncicos deberían ser considerados. Así, el promedio de erupción de dientes incluidos post exodoncia del supernumerario es 11 meses aproximadamente (4).

En lo referido al tiempo quirúrgico, algunos de los factores que podrían influenciarla serían la cantidad y tipo de supernumerarios, distancia al diente permanente no erupcionado y la localización del mismo (estructuras anatómicas adyacentes) (8), además de las complicaciones de cualquier procedimiento quirúrgico.

\section{CASO CLÍNICO}

Paciente, sexo masculino, de 11 años de edad llega derivado a la oficina de Cirugía Oral del Consultorio
Miraflores de Temuco por presentar dolor en la zona correspondiente a la pieza 1.1; el paciente no presentaba patologías sistémicas y aportaba historia odontológica previa sin complicaciones. En la anamnesis familiar el padre del paciente (43 años de edad) relata que hace 1 año él se sometió a cirugía oral para la remoción de un diente supernumerario en su zona anterosuperior. Clínicamente en el paciente no se encontró signos de alguna patología oral o extraoral (Figura 1). Se solicita una radiografía periapical céntrica de los incisivos centrales superiores (Figura 2). El análisis radiográfico revela una estructura radiopaca en relación al ápice de la pieza 1.1; la estructura presentaba delimitación de tejidos dentarios haciéndola compatible con un mesiodens sin formación radicular completa; esta estructura es única, correspondiendo a "mesiodens single cónico sin formación radicular completa". Se solicita una radiografía excéntrica para evaluar su posición (Figura 3) y una radiografía lateral para evaluar su nivel con respecto a la espina nasal anterior (Figura 4).

La dificultad de la intervención se presenta en las zonas anatómicas adyacentes al diente supernumerario. En la reunión clínica de especialidades odontológicas se planifica la cirugía inmediata. La clasificación del diente supernumerario indica pocas complicaciones de remoción quirúrgica.

En el procedimiento se utilizó anestesia local con un tubo de lidocaína al $2 \%$ con vasoconstrictor. Se realiza un colgajo de espesor completo vestibular amplio que permita una correcta visión del sitio y se localiza lo ubicación probable del mesiodens; se realiza una os-

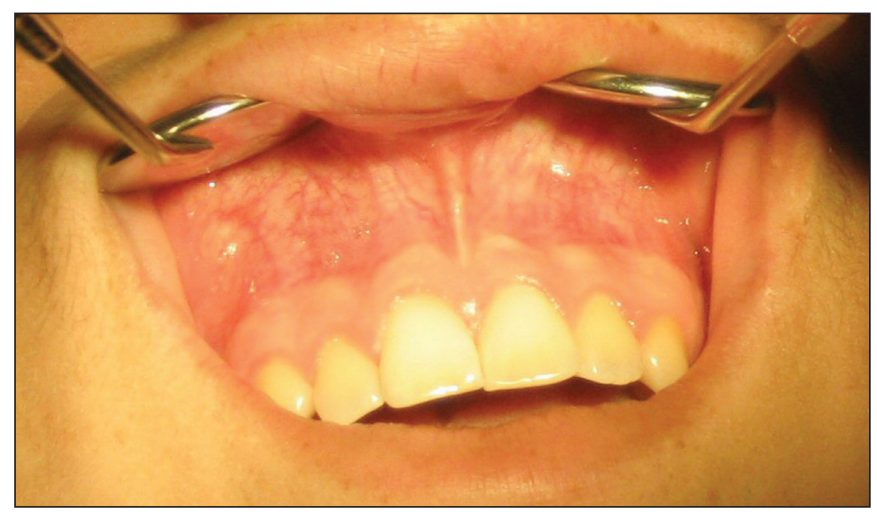

Fig. 1. 


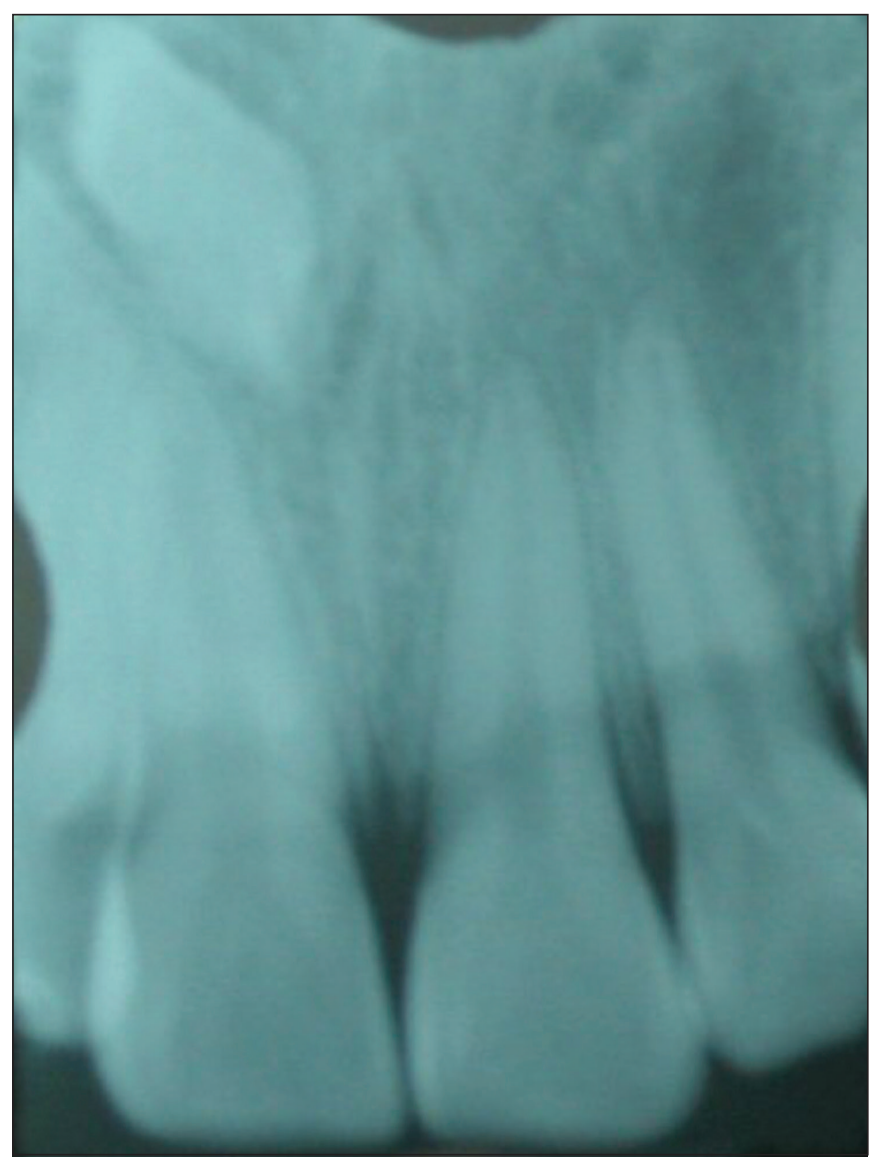

Fig. 2.

teotomía con baja velocidad e irrigación profusa cuidando no invadir la base de la espina nasal anterior y no llegar al ápice de la pieza 1.1. Se localiza el diente supernumerario y se realiza un contorneo con la misma fresa redonda de carbide a baja velocidad a fin de obtener un mejor acceso al sitio (Figura 5).

Con elevador fino angulado se accede al sitio y se logra luxar el mesiodens; la inclinación de este hizo aumentar la complicación operatoria y se decide realizar odontosección para facilitar la remoción del mesiodens (Figura 5), sin alterar el incisivo central. Se extrae el mesiodens (Figura 6) y se sutura con seda 3-0 y se indica terapia farmacológica con amoxicilina e ibuprofeno oral, además de las medidas inmediatas como la incorporación de hielo local, reposo y dieta blanda fría.

Las evaluaciones clínicas y radiográficas posteriores revelaron la ausencia de alteraciones en las estructu-

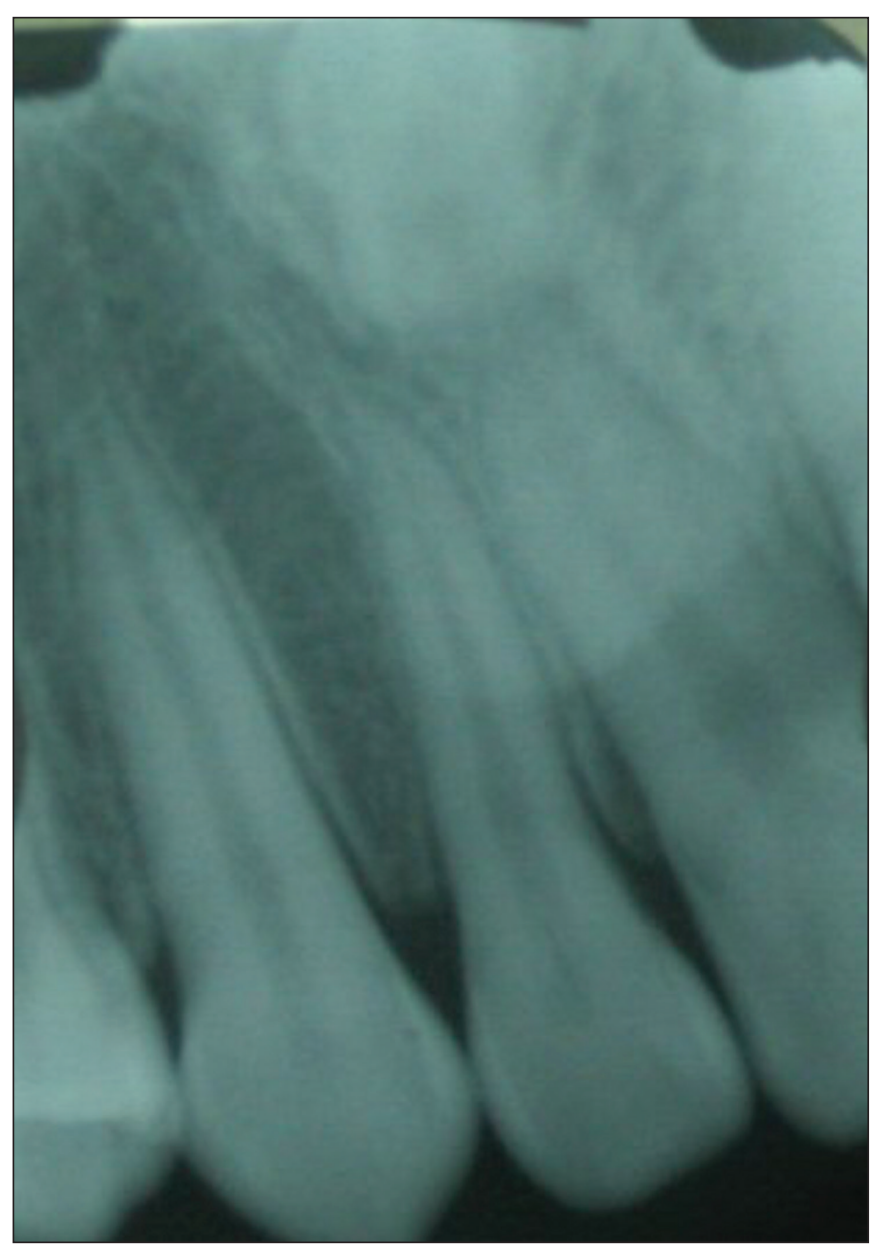

Fig. 3.

ras anatómicas adyacentes así como también una correcta cicatrización de los tejidos blandos (Figura 7).

El pronóstico es bueno. La principal complicación es la vitalidad de la pieza 1.1 , la que en el primer y segundo control no reflejo complicaciones; sin embargo, los controles y evaluaciones rutinarias son necesarios. El paciente y su familia siempre se mostraron cooperadores con sus tratamientos pasados, de modo que asumir y cumplir estos controles es fácil para ellos.

\section{CONCLUSIÓN}

Todo procedimiento médico debe estar protocolizado para que exista mayor predictibilidad de los resultados. Las reuniones clínicas frecuentes realizadas 


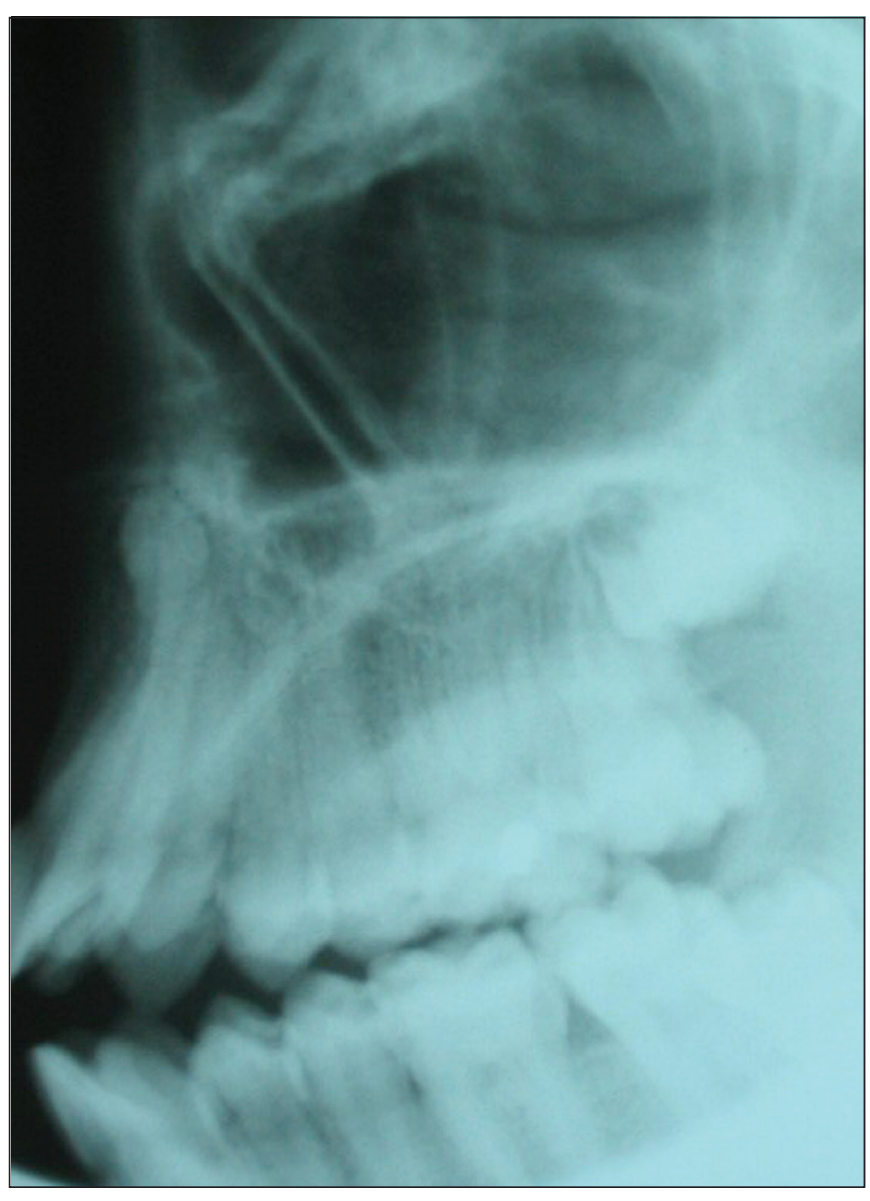

Fig. 4.

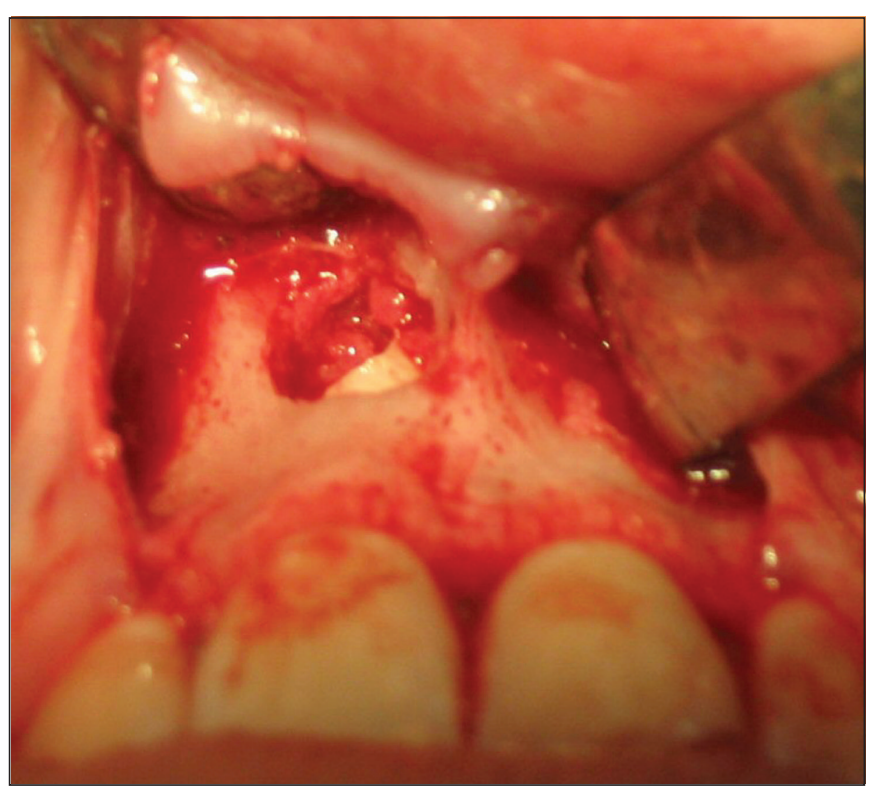

Fig. 5.

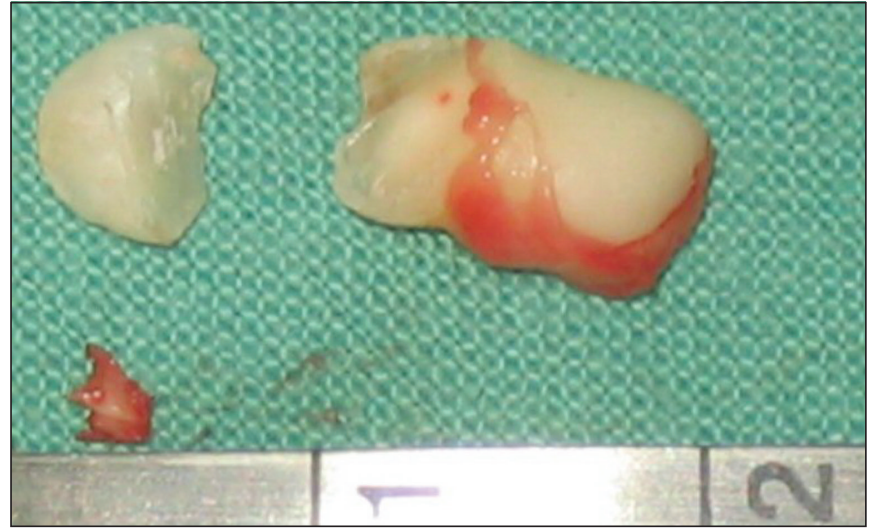

Fig. 6.

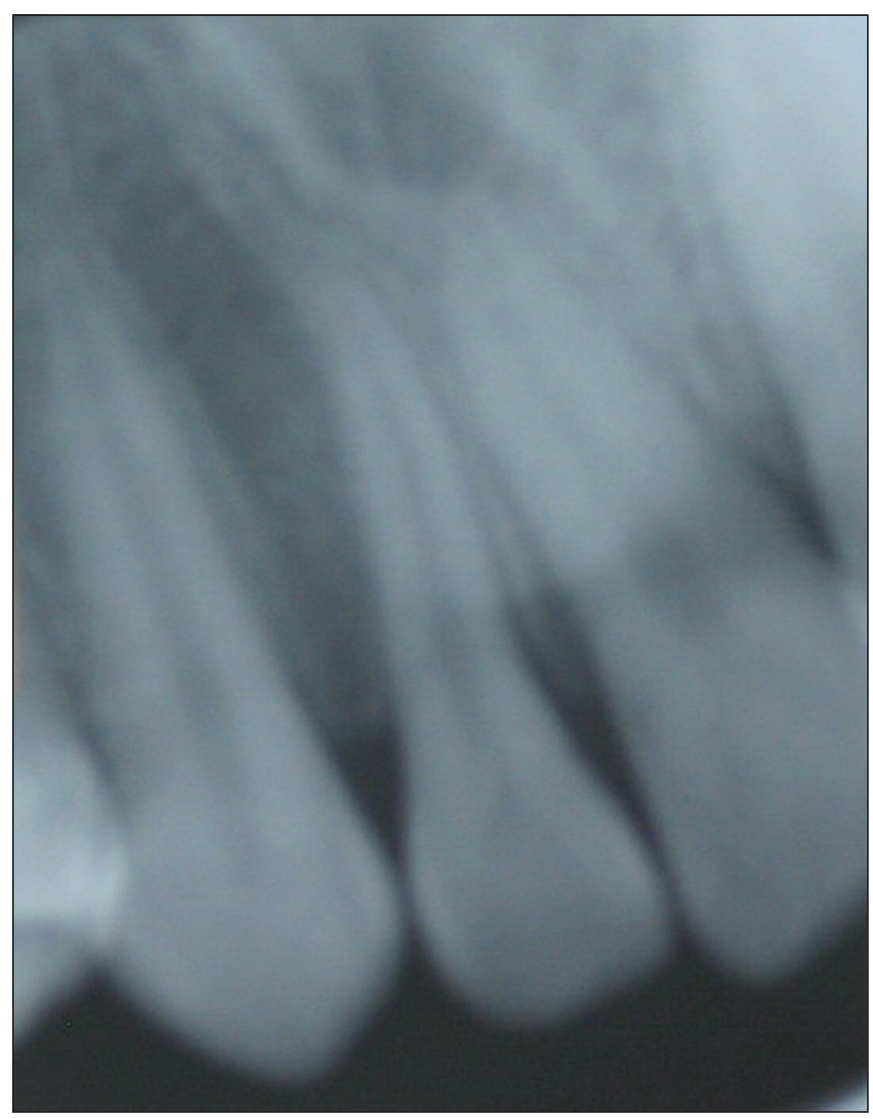

Fig. 7.

por nuestro equipo apuntan en tal dirección. La ausencia en la literatura de protocolos para el manejo de dientes supernumerarios hace necesario presentar nuestra experiencia para la discusión de la comunidad científica. No todos los casos se deben inter- 
venir quirúrgicamente, y cuando esta se realiza se deben evaluar situaciones como la ubicación, morfología, cantidad de piezas, tipo de dentición, además de la edad y colaboración del paciente y su familia.

Apoyados en la evidencia científica, los casos que hemos evaluado con este protocolo han sido exitosos en su manejo y los resultados han demostrado la satisfacción de nuestros pacientes.

\section{BIBLIOGRAFÍA}

1. Ripamonti U, Petit J, Thackeray J. A supernumerary tooth in a 1.7 million -year- old Australopithecus robustus from Swartkrans, South Africa. European Journal of Oral Science 1999;107: 317-21.

2. Bäckman B, Wahlin B. Variations in number and morphology of permanent teeth in 7-year-old swedish children. International journal of Paediatric Dentistry 2001;11:11-7.

3. Patchett CL, Crawford PJM, Cameron AC, Stephens $C D$. The management of supernumerary teeth in childhood - a retrospective study of practice in Bristol Dental Hospital, England and Westmead Dental Hospital, Sydney, Australia International Journal of Paediatric Dentistry 2001;11:259-65.

4. Mason C, Azam N, Rules DC. A retrospective study of unerupted maxillary incisors associated with supernumerary teeth. British Journal of Oral E Maxillofacial Surgery 2000;38:62-5.

5. Martins S, Tostes D, William E. Multiple dental anomalies in the maxillary incisor region. Journal of Orall Science 2003;47:47-50.

6. Betts A, Camilleri G, A review of 47 cases of unerupted maxillary incisors. International Journal of Pediatric Dentistry 1999;9:285-92.

7. Dalben GS, Gomide MR, Costa B, Neves LT. Description of a clinical technique for tooth extraction in the cleft lip and palate area. International Journal of Pediatric Dentistry 2001;11: 143-6.

8. Garvey T, Barry H, Blake M. Supernumerary teeth - an overview of classification, diagnosis and management. Journal of the Canadian Dental Association 1999;65:612-6.

9. Russell K, Folwarczna M. Mesiodens-diagnosis and management of a common supernumerary tooth. Journal of the Canadian Dental Association 2003;69 (6):362-6.

10. Jacobs S. Radiographic localization of unerupted teeth:Further findings about the vertical tube shift method and other localization techniques. American Journals Orthodontics \& Dentofacial Orthopedic 2000;118:439-47.

11. Koch H, Schwarz O, Klausen B. Indications for surgical removal of supernumerary teeth in the premaxilla. International Journal of Oral $\varepsilon$ Maxillofacial Surgery 1986;15:273-81.

12. Noguchi $M$, Tomizawa $M$, Suzuki $M$, Noda $T$. Impacted supernumerary tooth Developer Ander palatal polyp. International Journal of Paediatric Dentistry 2002;12:281-5.

13. Ebsan D, Tu H, Camarata J. Mandibular supernumerary tooth cusing neurosensory changes:a case report. Journal of Oral $\&$ Maxillofacial Surgery 2000;58:1450-1.

\section{CORRESPONDENCIA}

Sergio Olate Morales

Departamento de Odontología Integral

Facultad de Medicina

Universidad de La Frontera, Manuel Montt 112, Piso $4^{\circ}$.

Temuco, Chile

eMail: solate@ufro.cl 